\title{
FORUM
}

\section{Von der Griechenlandkrise zum Zerfall der Eurozone?}

\author{
Paul J.J. Welfens*
}

Im April 2010 ergab sich eine dramatische Verschärfung der Finanzierungsprobleme Griechenlands, das sich auf den Märkten immer höheren Zinsaufschlägen gegenüber sah: Eine Refinanzierung der Staatsschuld beziehungsweise die Finanzierung neuer Haushaltsdefizite war kaum noch darstellbar - zu groß war der Vertrauensverlust auf den internationalen Finanzmärkten. Der Zinsaufschlag bei langfristigen Staatsanleihen lag zeitweise mehr als 5 Prozentpunkte über der Zinsmarke für deutsche langfristige Staatsanleihen, die Anfang 2010 bei kaum 3 Prozent stand. Nachdem man mit einem Rettungspaket für Griechenland mehrere Wochen zögerte - gerade auch in Berlin -, wurde dann im Zuge der Verschärfung der Griechenlandkrise ein Rettungspaket unvermeidlich. Die Griechenlandkrise führte nicht nur zu einer hohen Volatilität der Kurse für griechische Staatsanleihen, sondern es ergaben sich auch Ansteckungs- beziehungsweise Regionalisierungseffekte. Das Misstrauen der internationalen Anleger beziehungsweise Spekulanten richtete sich Anfang Mai 2010 auch massiv gegen andere Länder der Eurozone mit hohen Haushaltsdefiziten und strukturellen Schwächen in der internationalen Wettbewerbsfähigkeit. Es ist erstaunlich, mit wie wenig Realitätssinn die Wirtschaftspolitik der Euro-Länder die Staatsfinanzkrise in den ,Club-Med-Ländern' im Mai 2010 angeht. Auf den internationalen Anleihemärkten droht ein Käuferstreik gegenüber Staatsanleihen aus Griechenland, Portugal, Spanien und Irland. In einer solchen Situation hat die Gruppe der Euro-Länder zusammen mit dem Internationalen Währungsfonds (IWF) für Griechenland ein dreijähriges Hilfspaket im Umfang von 110 Milliarden Euro auf den Weg gebracht, das gerade einmal einem Drittel der griechischen Staatsschuld entspricht. Das ist völlig unzureichend und es ist unverantwortlich, im Vergleich zu den globalisierten Finanzmärkten mit ihren großen Volumina eine solch geringe Garantiesumme zur Verfügung zu stellen. Dass 15 nationale Parlamente über größenordnungsmäßig relativ zum Bruttoinlandsprodukt der Eurozone geringfügige Garantien zeitraubend abstimmen müssen, kann man bei Griechenland vielleicht noch verteidigen. Diese Methode ist als allgemeiner Krisenmechanismus unbrauchbar. Die nächtlichen Sitzungen in Brüssel sind zudem technisch beziehungsweise inhaltlich nicht vernünftig vorbereitet und können von daher keine ideale Antwort auf die derzeitigen großen historischen Herausforderungen sein.

Mit einem großdimensionierten Hilfsrahmen von 750 Milliarden Euro - davon 250 Milliarden durch den IWF - hat die Eurozone Anfang Mai 2010 einen großen finanzpolitischen ,Airbag ' für Länder mit Finanzierungsproblemen aufgespannt. Diese Maßnahme signalisiert Entschlossenheit, Mitgliedsländer der Eurozone gegen internationale Spekulation zu verteidigen. Gleichwohl werden hiermit nur vorübergehende Stabilitätsimpulse erzeugt. Die Frage, wie die kritischen Länder der Eurozone ihre Schuldenquote mittelfristig reduzieren können, bleibt offen. Zwar kann man argumentieren, dass die Länder der Eurozone eine geringere Schuldenquote als die USA haben, dennoch ist die Eurozone strukturell beziehungsweise wirtschaftspolitisch schwach aufgestellt, da der Stabilitäts- und Wachstumspakt in der

Prof. Dr. Paul J.J. Welfens, Jean Monnet Professor für Europäische Wirtschaftsintegration, Lehrstuhl für Makroökonomik an der Bergischen Universität Wuppertal; Präsident des Europäischen Instituts für Internationale Wirtschaftsbeziehungen e.V. an der Bergischen Universität Wuppertal. 
ersten Dekade der Eurozone nicht wirklich zur Anwendung kam. ${ }^{1}$ Dies liegt nicht nur an einer unzureichenden Durchsetzung von Seiten der Europäischen Kommission, sondern bekanntlich haben auch Stabilitätssünder wie Deutschland und Frankreich eine Aufweichung des Paktes zu verantworten: Anstatt mit gutem Beispiel voranzugehen, haben beide Länder für eine Aufweichung des Paktes gesorgt.

\section{Gangbare Stabilisierungsoptionen}

Welche Optionen sind in einer akuten, dramatischen Situation mit einem sichtbaren Vertrauensverlust auf den Märkten und denkbarer Abwertungsspekulation gegen den Euro zu bedenken und welche Stabilisierungsansätze sind wirklich gangbar?

Bei der Suche nach einer Antwort sind vier Fakten in Rechnung zu stellen: 1) Die transatlantische Bankenkrise hat die Schuldenquoten der USA, Großbritanniens und der Eurozone binnen drei Jahren um rund 20 Prozentpunkte steigen lassen, das ist ein Anstieg um etwa ein Viertel und markiert einen dramatisch verminderten Handlungsspielraum der Staaten.

2) Bei einem verschlechterten Rating von Staatsanleihen, und zwar unterhalb von ,investment grade', werden Pensionsfonds und Versicherungen gezwungen, die betreffenden Anleihen mit Verlust zu verkaufen. Dann bleiben nur noch Banken - und unregulierte Hedgefonds -, die Staatsanleihen der ,Club-Med-Länder' halten können. Dennoch werden Banken auf mittlere Frist den Anteil dieser Anleihen in ihrem Portfolio reduzieren müssen, wenn sich die bislang hohe Kursvolatilität fortsetzt: Denn ein vernünftiges Risikomanagement erfordert dies und ein solches Management verlangen die Regierungen ja gerade verstärkt seit der transatlantischen Bankenkrise.

3) Eine massive Abwertung des Euros bedeutet faktisch eine Aufwertung des US-Dollars und erschwert damit, den aus US-Sicht notwendigen Abbau des hohen US-amerikanischen Leistungsbilanzdefizits zu erreichen. Wenn aus der Eurozone als zweitgrößtem Wirtschaftsblock der Welt Kapital massiv abfließt, dann wird es außer den USA vor allem einigen Schwellenländern zufließen, die sich zum Teil mit Kapitalverkehrskontrollen den Zuflüssen entgegenstellen werden, um das Entstehen spekulativer Blasen zu verhindern. Zugleich bedeutet eine Euro-Abwertung auch, dass die Abwertungen in den osteuropäischen EU-Mitgliedstaaten, die noch nicht der Eurozone angehören, noch größer sein werden, denn risikoscheue Anleger werden Kapital aus den schwer kalkulierbaren osteuropäischen Ländern abziehen. Dem werden sich die Länder teilweise mit Zinserhöhungen, die zu Investitionsund Wachstumsschwäche führen, entgegenstemmen.

4) Die neue Praxis der Europäischen Zentralbank (EZB), auch von Ländern mit schlechtem Rating Staatsanleihen über den Sekundärmarkt anzukaufen, kann man verteidigen. Es ist vorstellbar, dass die Zentralbank - ähnlich wie in den USA - Staatsanleihen im Portfolio hält, um hiermit auch das geldpolitische Instrumentarium zu erweitern. Problematisch wäre allerdings, wenn die EZB auch außerhalb einer extremen Marktsituation gerade Anleihen von Ländern mit schwachem Rating ankaufte. Eine klügere Strategie wäre es, wenn die EZB erklärte, längerfristig nur Anleihen von solchen Ländern der Eurozone anzukaufen, die den Stabilitäts- und Wachstumspakt längerfristig erfüllt haben. Es ist einerseits wichtig, dass EU-Mitgliedstaaten einander in schwierigen Zeiten helfen, andererseits sind langfristige Anreizwirkungen zu bedenken: Es gilt alle Länder zu ermutigen, sich stärker für nachhaltige Staatsfinanzen einzusetzen.

1 Martin Keim: Finanzmarktintegration in Europa: Implikationen für Stabilität und Wachstum in Sozialen Marktwirtschaften, Stuttgart 2009. 
Die finanzpolitische Situation von Griechenland und anderen ,Club-Med-Ländern “ war vor dem Start der Eurozone labiler als in 2010, sodass die scharfe Reaktion einiger Ratingagenturen beziehungsweise von Finanzmarkt-Akteuren teilweise als übertrieben erscheint. Länder wie Griechenland und Italien hatten Mitte der 1990er Jahre Zinsausgaben, die 11 Prozent des Bruttoinlandsproduktes erreichten; demgegenüber sind 6 Prozent in Griechenland in 2009 und 5 Prozent in Italien eine deutliche Verbesserung, sodass von einer Insolvenzgefährdung keine Rede sein kann. Es besteht allerdings ein Liquiditätsrisiko, denn die genannten Staaten, aber auch Portugal - bei einer Zinsausgabenquote von 3,5 Prozent in 2009 - und Irland könnten Probleme bei der marktmäßigen Finanzierung erleben, zumal beide Länder eine hohe Auslandsverschuldung haben.

Bei der Verabschiedung des Maastrichter Vertrages hat man die Dynamik der Finanzmarktglobalisierung einerseits und die Problematik des Stabilitäts- und Wachstumspaktes andererseits nicht ausreichend bedacht. Mit Blick auf den Pakt ist es unerlässlich, finanzpolitische Vernunft der Euro-Länder einzufordern: Hierzu ist zunächst eine neue Regelung notwendig, wonach Euro-Länder in einer Boomphase Haushaltsüberschüsse erzielen müssen. Diese neue Regel müsste mit automatischen Sanktionen beziehungsweise Strafzahlungen versehen sein und dies ließe sich in einer Boomphase auch tatsächlich durchsetzen. Es ist bezeichnend, dass ein Land wie Griechenland in einer Dekade Euro-Mitgliedschaft trotz mehrerer Jahre mit Wachstumsraten von 3 bis 4 Prozent kein einziges Mal einen Haushaltsüberschuss erzielt hat. Daher ist die bisherige Anreizstruktur des Stabilitäts- und Wachstumspaktes fehlerhaft. Man wird realistischerweise mit Blick auf die relativ labile globale Finanzdynamik zusätzliche Reformmöglichkeiten betrachten müssen. Unter der Bedingung, dass alle Mitgliedsländer deutliche Konsolidierungserfolge erzielen, ist an eine EU-Schuldenagentur zu denken, wobei im Vorfeld eine verstärkte wirtschaftspolitische Koordinierung zu verankern wäre. Dazu könnte auch gehören, dass die Europäische Kommission und das betreffende Mitgliedsland im zweijährigen Rhythmus einen Bericht zur finanzpolitischen Nachhaltigkeit (inklusive Vergleichszahlen für alle EU-Mitgliedstaaten) vorlegen, der allen Bürgerinnen und Bürgern als Kurzbericht zuzuleiten wäre. Dies soll der Entwicklung einer gemeinschaftlichen Stabilitätskultur in der Eurozone dienen. Durch die Einrichtung einer EU-Schuldenagentur, bei der Gemeinschaftsanleihen für alle Länder der Eurozone aufgelegt werden - soweit hierfür die emittierenden Staaten Sicherheiten (etwa bei Infrastrukturprojekten) stellen können - kann die Vertrauenskrise an den Märkten überwunden werden. Allerdings müssen dabei die Regeln des Stabilitäts- und Wachstumspakts in der Europäischen Union verschärft werden. Zudem ist der IWF grundsätzlich in die Stabilisierung einzubeziehen.

Es wird höchste Zeit, die Stabilisierung des Euros als ein internationales öffentliches Gut der OECD-Länder zu definieren: Wenn es zu einer massiven Destabilisierung der Eurozone käme und Länder wie Spanien oder Italien die übliche Refinanzierung der Staatsschuld infolge sehr hoher Zinsaufschläge nicht mehr am internationalen Kapitalmarkt darstellen könnten, kämen auch Japan und - vermutlich mit Verzögerung - die USA und Großbritannien in Schwierigkeiten. Auf dem Fuß folgte dann wegen neuer riesiger Abschreibungen bei Wertpapieren eine ernste globale Bankenkrise. Eine gefährliche neue Weltrezession wäre die Folge.

Bisher mangelt es auf Seiten der Politik an der Fähigkeit, Lösungen vernünftig zu dimensionieren und die komplizierten Probleme auf den Weltfinanzmärkten beziehungsweise die eigenen Politikoptionen zu erklären. Zugleich ist mit Bedauern festzustellen, wie wenige Reformen die Politik bislang in Sachen Finanzmarktaufsicht wirklich beschlossen hat. Die- 
ser Vorwurf ist an fast alle OECD-Länder zu richten, wo man sich mit Blick auf positive Wachstumsraten für 2010 allzu früh von notwendigen Reformen zurückgezogen hat.

Im Übrigen kann man die Ratings bei Griechenland-Anleihen kritisieren, aber die viel zu langsam in Sachen Finanzmarktregulierung handelnde Europäische Kommission hat es auch zwei Jahre nach der Bankenkrise nicht geschafft, eine EU-Rating-Agentur auf den Weg zu bringen, die den Wettbewerb auf dem Rating-Markt sinnvoll intensiviert. Wenn jetzt - mitten in der Griechenlandkrise - eine solche Rating-Agentur eingerichtet werden soll, wird man den Verdacht haben, dass hier eine ,Beschönigungsagentur' auf den Weg gebracht werden soll. Eine EU-Rating-Agentur ist aber in der Tat im Interesse von mehr Wettbewerb auf dem Rating-Markt notwendig, wobei man auf politische Unabhängigkeit größten Wert legen sollte. Eine Stiftungslösung mit wissenschaftlicher Fundierung ist empfehlenswert. Wenn die Eurozone zusammen mit den USA und Großbritannien keine Stabilisierung der Märkte erreichen sollte, dann steht nicht nur der Euro vor dem Ende, sondern die Europäische Union wird zerfallen. Daran kann niemand in den OECD-Ländern ein Interesse haben. Die Schuldenquote der Eurozone dürfte 2010 - nach EU-Prognosen - 85 Prozent erreichen, 94 Prozent in den USA und 79 Prozent in Großbritannien, das binnen vier Jahren fast eine Verdopplung der Schuldenquote realisiert hat. Bei genauerem Hinsehen ist die Eurozone als Ganzes hinsichtlich der Schuldenquote stabiler aufgestellt als die USA es sind, für die 2011 schon eine Schuldenquote von 103 Prozent prognostiziert wird.

Was die Befürchtungen hinsichtlich eines starken Inflationsdrucks wegen der Staatsfinanzkrise in der Eurozone angeht, so ist darauf hinzuweisen, dass die EZB auch bei einem Ankauf von Staatsanleihen in größerem Umfang über Sterilisierungsmaßnahmen ${ }^{2}$ die Kontrolle der Geldmenge in stabilitätsförderlicher Weise gewährleisten kann. Ein echter Problemdruck entsteht nur, wenn mittelfristig tatsächlich ein Land der Eurozone seine Kredite nicht zurückzahlen kann. Die EZB müsste dann bilanzmäßig die Verluste im Wesentlichen monetarisieren, also eine ungeplante Ausweitung der Geldmenge vornehmen. Von einer solchen Situation ist man in der Eurozone zunächst einmal wohl für einige Jahre entfernt. Zugleich wird hier deutlich, dass man unrealistische Konsolidierungspläne, wie sie im Fall Griechenlands auf den Weg gebracht wurden, unbedingt unterlassen sollte. Griechenlands Anpassungsfähigkeit wird wegen des zu kurzen Drei-Jahres-Zeitraums überfordert; hier sollte man unbedingt sorgfältig eine veränderte Anpassungsplanung vornehmen. Das Kanzleramt ist in der Griechenland- beziehungsweise Eurokrise erkennbar schwach in der wirtschaftspolitischen Beratung aufgestellt: Obwohl dem Hauptberater der Kanzlerin Szenarioanalysen zu einer Griechenland/Eurokrise bekannt waren, hat man keine vorsorglichen Pläne entwickelt.

War die Griechenlandkrise abzusehen? Antwort ,Ja': Bereits im Herbst 2008 wies der Autor, mit Blick auf die Perspektiven der Bankenkrise darauf hin, dass die Eurozone in ernste Schwierigkeiten geraten könnte, wenn die Risikoprämien für Länder wie Griechenland, Italien, Spanien oder Portugal stark ansteigen. ${ }^{3}$ Es ist mit Blick auf Griechenland und Italien als Ländern mit hoher Schuldenquote und hoher Defizitquote sowie hohen Auslandsschulden keineswegs ausgeschlossen, dass bei einer temporären Verschärfung der globalen Finanzkrise eine marktmäßige Refinanzierung nicht mehr darstellbar ist - wenn indes etwa ein Land wie Griechenland im Wesentlichen durch die Impulse der US-Bankenkrise in ernste Probleme kommt, so sollte man die ,No-bail-out'-Vorgaben aus dem Maastrichter

2 Bei Sterilisierungsmaßnahmen nimmt die EZB über Ausgleichsmaßnahmen bei den Banken soviel Liquidität weg, wie sie durch den Ankauf von Staatsanleihen zuvor geschaffen hatte.

3 Paul J.J. Welfens: Transatlantische Bankenkrise, Stuttgart 2009, S. 158-159. 
Vertrag nicht zur Anwendung bringen. Vielmehr sollten die Mitgliedsländer der Eurozone sich in solidarischer Verantwortung auch hinter Mitgliedsländer mit Refinanzierungsproblemen stellen. So wie im Rahmen von ,Mega'-Hilfspaketen EU-Mitgliedstaaten Garantien für eigene Banken angeboten haben, so sollte man auch Garantiepakete von Seiten mehrerer EU-Mitgliedstaaten für Euro-Länder mit besonderen Refinanzierungsproblemen geben. Ob die Europäische Investitionsbank als EU-Institution hier auch besondere Garantien für einige Jahre geben könnte, bleibt zu überlegen. Man sollte jedenfalls in einer globalen Finanzkrise nicht die für eine normale Finanzwelt gedachten Regeln aus dem Maastrichter Vertrag anwenden. Das heißt allerdings nun nicht, dass EU-Mitgliedstaaten sich gegenseitig eine Freifahrkarte für laxe Fiskalpolitik und hohe Defizitquoten ausstellen sollen. Nachdem sich in der transatlantischen Bankenkrise gerade die Währungsintegration beziehungsweise die Eurozone bewährt hat, wäre es aber gänzlich unvernünftig, die Wirtschafts- und Währungsunion durch eine überzogene Interpretation des Maastrichter Vertrags in Schwierigkeiten zu bringen. Die Eurozone dürfte im Übrigen an Attraktivität gewonnen haben. Ein baldiger Beitritt zur Eurozone dürfte für viele osteuropäische EU-Mitgliedstaaten zu einem mittelfristig gewichtigen Ziel werden, denn eine Mitgliedschaft in der Eurozone bietet bei internationalen beziehungsweise globalen Finanzmarktinstabilitäten offenbar einen gewissen Schutz. Die Eurozone beziehungsweise die Europäische Union wird aber gut daran tun, die Konvergenzkriterien nicht aufzuweichen und ergänzend auch die Leistungsbilanzdefizitquote von Kandidatenländern kritisch zu beachten.

Es ist wichtig, einen kritischen Blick auf die Ansatzpunkte des Ausgangspunkts der Krisedynamik zu lenken, nämlich die Griechenlandkrise, deren Mechanismen im Übrigen durchaus mit der herkömmlichen Lehrbuchliteratur erklärt werden können. ${ }^{4}$

\section{Griechenland-Hilfe: falsche Bankenkritik gefährdet die EU}

110 Milliarden Euro von den Ländern der Eurozone und vom IWF sollen Griechenland gemäß der Beschlüsse vom 8. Mai 2010 im Rahmen eines Drei-Jahres-Hilfspakets stabilisieren. Dieses Paket ist völlig unzureichend dimensioniert und zugleich werden die Banken von der Politik beschimpft, deren Kauf von Staatsanleihen der EU-Mitgliedstaaten für Stabilität dringlich ist. In einer Krisenphase auf den Märkten, in denen die Politik durch Zögerlichkeit Vertrauenskapital verspielt und die Kurse der Staatsanleihen vieler Länder mit unter Druck gesetzt hat, ist die populäre Bankenbeschimpfung töricht. Das passt alles nicht zusammen und mündet in einen Käuferstreik gegenüber Anleihen der ,Club-Med-Länder', was wiederum den Euro abstürzen lassen könnte.

Griechenland-Anleihen sind nach dem durch „Standard \& Poor's“ erfolgten Heruntersetzen des Ratings auf Nicht-, investment grade“ Mitte April 2010 aus den Depots der großen Pensionsfonds verschwunden, die aus regulatorischen Gründen hierzu gezwungen waren und dabei kräftige Verluste gemacht haben. Entsprechend dem Kursverfall bei Griechenland-Anleihen gehen viele Fonds bei diesen mit 25 Prozent Verlust aus der jeweiligen Position. Pensionsfonds und ähnlich regulierte Versicherungen haben weltweit fast kein Interesse an Anleihen der, Club-Med-Länder'. Auch Banken mussten für das erste Quartal 2010 Verluste bei Griechenland-Anleihen bilanzieren, eine Chance auf Zuschreibungen bestand immerhin.

Eigentlich müssten viele Banken wegen der hohen Volatilität der Griechenland-Anleihen unter dem Aspekt des Risikomanagements diese Anleihen verkaufen. Ähnliches gilt für an-

4 Paul J.J. Welfens: Grundlagen der Wirtschaftspolitik, 4. Auflage, Heidelberg 2010. 
dere Staatsanleihen der ,Club-Med-Länder' mit zeitweise hohen ,spreads ' beziehungsweise starken Kursschwankungen. Bevor die Politik laut darüber nachdenkt, die Banken bei einer ,Griechenland-Hilfsaktion“ stark zur Kasse zu bitten beziehungsweise weiter zu beschimpfen, sollte sie sich zunächst klar machen, dass das Halten von Griechenland-Anleihen schon ein halbes Geschenk der Banken an den europäischen Gemeinsinn darstellt.

Zugleich besteht ein gewisser Widerspruch zu einer der offiziellen Schlussfolgerungen der Bankenkrise 2007 bis 2009, wonach ein verbessertes Risikomanagement unerlässlich ist. Ein vernünftiges Risikomanagement verlangt eben den Verkauf von volatilen Papieren der ,Club-Med-Länder'. Hier ist also ein ernster Widerspruch entstanden, den man auf Dauer nicht ungestraft beibehalten kann. Wenn Regierungen der EU-Mitgliedstaaten ihre jeweiligen Banken bedrängen, unbedingt weiterhin Papiere der ,Club-Med-Länder ${ }^{\star}$ zu halten und Kreditlinien fortzuführen, so klingt das widersprüchlich. Anleihen mit schlechten Ratings zu halten, bedeutet für Banken zudem, dass die regulatorischen Eigenkapitalanforderungen gemäß den ,Basel-II ${ }^{6}-R^{-R e g e l n}{ }^{5}$ ansteigen beziehungsweise der Kreditvergabespielraum für die Wirtschaft sinkt. Es ist aber kaum sinnvoll, Banken aufzufordern, Staatskredite verstärkt an ein Land mit einem schlechten Rating zu geben und zugleich gegenüber den Banken zu klagen, sie gäben zu wenige Kredite an die Privatwirtschaft - was den Aufschwung behindere. Man sieht schon hier, dass die ,Basel-II'-Regeln dringend überarbeitet werden müssen.

Wenn man betrachtet, dass der Bund für die „Hypo Real Estate“ mit über 100 Milliarden Euro gebürgt hat, so ist klar, dass ein Paket im Umfang von 110 Milliarden Euro für Griechenland beziehungsweise entsprechende staatliche Bürgschaften (Deutschland bürgt mit 22,4 Milliarden Euro) völlig unterdimensioniert ist. Den Bürgschaftsrahmen hätten die EUMitgliedstaaten - nicht die Euro-Länder - über den kompletten Bestand der griechischen Staatsschuld von 300 Milliarden Euro aufspannen sollen plus ein Sicherheitszuschlag von 30 Prozent; dann wäre man bei 390 Milliarden Euro. Ein breiter Garantieschirm ist die beste Versicherung, dass die bürgenden Staaten am Ende für die Bürgschaft nicht zur Kasse gebeten werden! Die zu schmal geschnittene Bürgschaft für Griechenland wiederum könnte Deutschland und die anderen Länder der Eurozone noch teuer zu stehen kommen. Bei so miserablem Krisenmanagement und Unverständnis in Teilen der Politik für die Dramatik der Lage in der Eurozone könnte diese mitsamt der Europäischen Union zerfallen.

Die in den nächsten Jahren anstehenden Refinanzierungen in Griechenland, Portugal, Spanien und Irland - Ländern mit einer Auslandsverschuldung von zum Teil deutlich über 50 Prozent des Bruttoinlandsprodukts in 2009 - werden kaum realisierbar sein, da in den Finanzmärkten eine massive Vertrauenskrise gegenüber Ländern der Eurozone entstanden ist. Diese ist teilweise ein Echo der transatlantischen Bankenkrise, in deren Verlauf die staatlichen Schuldenquoten in fast allen EU-Mitgliedstaaten deutlich angestiegen sind. Dies nährt vielfach ernste Befürchtungen über die Möglichkeiten einer nachhaltigen Finanzierbarkeit der Staatsverschuldung, vor allem bei Ländern mit hohen Schuldenquoten und hoher Auslandsverschuldung. Eine hohe Auslandsverschuldung bedeutet eine starke Abhängigkeit von Ratings. Eine Herunterstufung des Ratings bei Staaten führt zu deutlich erhöhten Risikoprämien und parallel dazu zu einem Absinken der Anleihenkurse. Erhöhte Zinssätze für Staaten bedeuten dann auch erhöhte Finanzierungskosten für Banken und dies wird die Investitions-

5 ,Basel II ‘ ist die umgangssprachliche Bezeichnung für eine Rahmenvereinbarung über die internationale Konvergenz der Kapitalmessung und Eigenkapitalanforderungen. Siehe: Baseler Ausschuss für Bankenaufsicht: Internationale Konvergenz der Kapitalmessung und Eigenkapitalanforderungen. Überarbeitete Rahmenvereinbarung, Juni 2004. 
finanzierung beziehungsweise das Wirtschaftswachstum und damit wiederum die staatliche Haushaltskonsolidierung erschweren.

\section{Maßnahmen zur Stabilisierung von Markt und Vertrauen}

Zur Vertrauens- und Marktstabilisierung sind folgende Schritte unerlässlich: Die Europäische Union sollte eine EU-Schuldenagentur gründen, die Staatsanleihen für Mitgliedstaaten ausgeben kann. Beim Stabilitäts- und Wachstumspakt ist eine sinnvolle Verschärfung dahin gehend notwendig, dass in Boomphasen zwingend Haushaltsüberschüsse zu realisieren sind. Wird dies verfehlt, erfolgt eine deutliche Kürzung von EU-Zuwendungen. Die Schaffung einer EU-Rating-Agentur ist umgehend auf den Weg zu bringen, die Europäische Union hat hier viel Zeit verloren.

Worin besteht konkret der Ausweg aus dem möglichen Zerfall der Eurozone und letztlich der Europäischen Union - ein drohendes Szenario für den Fall, dass die ,Club-Med-Länder ‘ gleichzeitig den Zugang zum Kapitalmarkt verlieren. Bevor eine solche Situation binnen weniger Jahre womöglich eintritt, sollte man dem Horrorszenario energisch entgegentreten:

- Durch die Schaffung einer sorgfältig konstruierten EU-Schuldenagentur, die auf Euro denominierte Wertpapiere für alle Mitgliedsländer der Eurozone am Markt platziert, kann man die Situation nachhaltig stabilisieren. Die Platzierung solcher Anleihen dürfte zu einer Abwertung der umlaufenden Papiere führen, sofern sie nicht von der Gemeinschaft der EU-Mitgliedstaaten - oder einer Gruppe von EU-Mitgliedstaaten - garantiert werden. Eine EU-Schuldenagentur kann man sich sinnvoll nur als Bestandteil einer stärker vergemeinschafteten Wirtschaftspolitik der Länder der Eurozone vorstellen.

- Notwendig ist eine Stärkung des Kapitalmarktvertrauens und das erfordert eine Ergänzung des Stabilitäts- und Wachstumspakts. Vor dem Hintergrund der bisherigen schlechten Erfahrungen mit dem Pakt sollte gelten: Länder der Eurozone sollten in Boomphasen zwingend einen mindestens einjährigen Budgetüberschuss erzielen. Bei einer solchen neuen Vorgabe vermeidet man in Verbindung mit der 3-Prozent-Obergrenze bei der Neuverschuldungsquote ein ewiges Ansteigen der Schuldenquote, wobei hier die 60-ProzentObergrenzen der Gesamtverschuldung längerfristig beizubehalten ist. Notwendig ist, dass bei Verletzung der Vorgaben eines zeitweiligen Haushaltsüberschusses automatisch eine Kürzung der EU-Mittelzuflüsse für das betreffende Land erfolgt. Die Kürzung von EUMitteln in einer Boomphase ist politisch durchsetzbar und setzt sinnvolle Anreize - anders als eine direkte Mittelkürzung in einer Rezessionsphase. Wird die 3-Prozent-Marke außerhalb einer schweren Rezession überschritten, so ist ebenfalls eine EU-Mittelkürzung sinnvoll, die allerdings erst in einer Boomphase vollzogen werden sollte. Schuldenbegrenzungen könnten in den Verfassungen der EU-Mitgliedstaaten verankert werden, sie sind stärker wirksam als einfachgesetzliche Begrenzungen. Allerdings hat eine strukturelle Einschränkung des Spielraums auf nationaler Ebene nur wirklich Sinn, wenn für starke Rezessionen fiskalpolitisch angemessene Expansionsspielräume auf nationaler Ebene oder auf supranationaler Ebene erhalten bleiben.

- Die Errichtung einer politisch unabhängigen EU-Rating-Agentur - zum Beispiel in der Rechtsform einer Stiftung - ist unerlässlich und seit Jahren überfällig. Mehr Wettbewerb auf dem Rating-Markt in Verbindung mit besseren Standards könnte deutlichere Kapitalmarktsignale setzen. Das wäre ein wichtiger Beitrag zu mehr Stabilität in der Europäischen Union und weltweit. Die Finanzierung der Rating-Agenturen ist dabei durch ein zweistufiges Pool-System zu ersetzen: Jeder Emittent zahlt nach Maßgabe des Marktanteils in einen Finanzierungspool ein, auf der zweiten Stufe erfolgt im Wettbewerb das Ra- 
ting. Das Rating bekommt damit die Qualität eines öffentlichen Gutes, wobei Fehlanreize vermieden werden. Im bisherigen System zahlt der jeweilige Emittent selbst , seiner ' Rating-Agentur ein Leistungsentgelt, was natürlich zu Fehlanreizen führt. Wenn hingegen alle Emittenten in der Europäischen Union in einen Finanzierungstopf einzahlten, auf deren Basis dann in einem von neutraler Seite organisierten Ausschreibungsverfahren jeweils einer Ratingagentur ein Ratingjob zugeordnet wird, ergäbe sich ein viel besseres System.

- Die Erhaltung der internationalen Wettbewerbsfähigkeit und damit das Vermeiden von anhaltenden massiven Leistungsbilanzdefiziten ist wesentlich, um ein unkontrolliertes Anwachsen der Auslandsverschuldung beziehungsweise der Staatsverschuldung in ausländischer Hand zu vermeiden. Die EU-Haushaltsüberwachung ist daher um ein Element des Kontrollierens von Leistungsbilanzdefiziten zu ergänzen. Alle Mitgliedsländer der Eurozone hätten hier künftig im Rahmen der vorzulegenden Konvergenzprogramme gemäß modifiziertem Stabilitäts- und Wachstumspakt - Anpassungsprogramme vorzulegen.

- Als sehr problematisch muss gelten, wenn - wie bislang weithin doch üblich - Kreditausfallversicherungen (Credit Default Swap, CDS) von Banken angeboten werden. CDS-Papiere sollten nur von einer klar vom Bankensektor abgegrenzten Versicherungswirtschaft angeboten werden dürfen. Denn sonst kann das ernste Problem entstehen, dass Banken Anleihen haben beziehungsweise am Markt platzieren, nachfolgend verkaufen und dabei zugleich durch Kauf von CDS-Papieren auf den Konkurs des Emittenten spekulieren. Erwerber von CDS-Papieren sollte im Übrigen nur sein, wer in entsprechendem Umfang die jeweiligen Anleihen besitzt.

Die Finanzmärkte sind endlich, gemäß den Zielsetzungen der G20, zu reformieren, wobei eine bessere Bankenaufsicht und ein sinnvolleres Rating-Geschäft zu fordern sind. Wenn die Europäische Union weiter im Schlafmützentempo über eine EU-Rating-Agentur diskutiert, wird die Europäische Union Geschichte sein, bevor die neue Agentur arbeitet. Zudem ist eine Volatilitätssteuer bezogen auf die Eigenkapitalrendite von Finanzmarktanbietern sinnvoll, damit diese einen längeren Zeithorizont annehmen, was mehr Stabilität bringt. ${ }^{6}$ Wenn die bislang zögerliche Europäische Kommission und die EU-Mitgliedstaaten nicht endlich eine realistische Politikstrategie im Umfeld einer historisch einmaligen internationalen Wirtschaftskrise entwickeln, werden Eurozone und Europäische Union auseinanderbrechen; mit sehr weitreichenden negativen Folgewirkungen für Politik und Wirtschaft in Europa beziehungsweise der ganzen Welt. Ein großes Fragezeichen steht über allen Ländern mit hoher staatlicher Schuldenquote und zugleich hohem Anteil an Auslandsschulden. Denn hier drohen bei einem internationalen Vertrauensverlust rasch hohe Kapitalabflüsse. International hat Japan die höchste Schuldenquote, nämlich rund 200 Prozent zu Beginn 2010, aber der Anteil der Auslandsschulden relativ zum japanischen Bruttoinlandsprodukt dürfte weniger als 10 Prozent betragen, wenn man Schätzungen der „Economist Intelligence Unit“ folgt. Von daher und in Anbetracht geringer langfristiger Zinssätze von rund 1,5 Prozent - die Zinsausgaben des Staates relativ zum Bruttoinlandsprodukt sind also unter 4 Prozent - ist Japan vorläufig als stabil einzuschätzen. Hingegen erreichten Griechenlands Zinsausgaben in 20096 Prozent des Bruttoinlandsprodukts.

6 Ulrich Heilemann/Paul J.J. Welfens (Hrsg.): Zukunftsfähige Wirtschaftspolitik in Deutschland und Europa, Heidelberg 2010. 
Aus Sicht der makroprudentiellen Aufsicht, die das Systemrisiko der Finanzmarktdynamik auf dem Analyseradar haben sollte, ist daher der Anteil der Papiere um den kritischen Bereich des ,investment grade'-Rating von großem Interesse. Von Seiten der Finanzmarktaufsicht wird diese kritische Größe aber bislang nicht erfasst - entsprechende Reformen sind notwendig. Die makroprudentielle Aufsicht ist aus wirtschaftspolitischer Sicht mit der mikroprudentiellen - auf einzelne Banken beziehungsweise Finanzmarktanbieter ausgerichteten - sinnvoll zu verbinden. Das ist insbesondere für kleine Volkswirtschaften unerlässlich, wo bei typischer Dominanz sehr weniger Finanzinstitute schon die Schieflage eines einzigen großen Anbieters ein Systemrisiko bedeuten kann. Wenn in einem Währungsraum wie der Eurozone die Ratings von Beständen von Anleihen einiger Mitgliedsländer stark abgewertet werden, so zeigt dies nicht nur Symptome einer Staatsfinanzierungskrise, sondern natürlich ist auch das Rating von Banken, die solche Papiere halten, von einer Herabstufung bedroht. Damit aber steigen dann die Kapitalkosten beziehungsweise droht dann zudem eine Abschwächung des Wirtschaftswachstums, was wiederum das Rating der betreffenden staatlichen Schuldverschreibung verschlechtern kann. Eigentlich bietet sich hier an, dass aus Gründen des Risikomanagements von Großbanken die Anteile von Papieren mit schlechten Ratings im eigenen Portfolio reduziert werden.

Mit Blick auf eine solche eigentlich vernünftige Verhaltensweise gab es jedoch im Mai 2010 erhebliche Konsistenzprobleme in der Eurozone, da die Politik in vielen Mitgliedsländern heimische Banken dazu drängte, Griechenland-Anleihen, deren Rating heruntergestuft wurde, im Bestand unverändert zu halten oder gar Papiere hinzuzukaufen. Allerdings gibt es auch Konsistenzprobleme bei den führenden Rating-Agenturen selbst, deren Ratings im Vorfeld der Krise vom Mai 2010 recht unterschiedlich bei zehnjährigen griechischen Staatsanleihen waren (siehe Tabelle 1).

Tabelle 1: Übersicht über die Entwicklung der Ratings für griechische Staatsanleihen mit einer Laufzeit von 10 Jahren

\begin{tabular}{|l|c|c|c|}
\hline \multicolumn{1}{|c|}{ Datum } & Fitch Ratings & Moody's & Standards \& Poor's \\
\hline 22. Oktober 2009 & A- & & \\
\hline 29. Oktober 2009 & & A1(-) & \\
\hline 07. Dezember 2009 & & & A-(-) \\
\hline 08. Dezember 2009 & BBB+ & & BBB+(-) \\
\hline 16. Dezember 2009 & & & \\
\hline 22. Dezember 2009 & & A2 & \\
\hline 09. April 2010 & BBB- & & BB+ \\
\hline 22. April 2010 & & A3(-) & \\
\hline 27. April 2010 & & Ba1 & \\
\hline 14. Juni 2010 & & & \\
\hline
\end{tabular}

Quelle: Eigene Darstellung auf Basis der Daten der drei genannten Rating-Agenturen. 
Die Europäische Kommission und die Mitgliedsländer der Eurozone sollten dringend realistische beziehungsweise mehr langfristige Anpassungsprogramme auf den Weg bringen. Das Vertrauen von Wählern einerseits und Marktteilnehmern andererseits lässt sich nur gewinnen, wenn eine realistische und zugleich ursachenadäquate Therapie vorgenommen wird. Dabei ist zu Berücksichtigen, dass etwa die Leistungspositionen der Länder der Eurozone recht unterschiedlich sind. ${ }^{7}$ Die massive Exportexpansionsstrategie Deutschlands ist im Übrigen eine Mitursache für hohe Leistungsbilanzdefizite von ,Club-Med-Ländern`. Dennoch kann nicht gefolgert werden, dass vor allem Deutschland Anpassungsmaßnahmen durchzuführen hat. Die wichtigsten Anpassungsschritte sind eindeutig von Ländern mit relativ schwacher internationaler Wettbewerbsfähigkeit durchzuführen. Hierbei könnte vernünftigerweise die Europäische Kommission mit einer Task-Force von Experten die Anpassungsschritte unterstützen. Die Europäische Union steht vor gefährlichen historischen Herausforderungen.

7 Europäische Kommission: The impact of the global crisis on competitiveness and current account divergences in the euro area, Quarterly Report on the Euro Area 1/2010. 